\title{
Concentrated Growth Factor from Autologous Platelet Promotes Hair Growth in Androgenetic Alopecia
}

\author{
Poh-Ching Tan, Yun Xie, Wei Wu, Peiqi Zhang, Yiming Gao, Kai Liu, Shuangbai Zhou, \\ Qingfeng Li
}

Department of Plastic \& Reconstructive Surgery, Shanghai Ninth People's Hospital, Shanghai Medical College of Jiao Tong University, Shanghai, China

Correspondence to: Shuangbai Zhou, shuangbaizhou@yahoo.com; Qingfeng Li, dr.liqingfeng@yahoo.com Keywords: Androgenetic Alopecia, Concentrated Growth Factor

Received: January 15, $2019 \quad$ Accepted: March 11, $2019 \quad$ Published: March 14, 2019

Copyright $\odot 2019$ by authors and Scientific Research Publishing Inc.

This work is licensed under the Creative Commons Attribution International License (CC BY 4.0).

http://creativecommons.org/licenses/by/4.0/

\section{(c) (1) Open Access}

\section{ABSTRACT}

Studies have shown that platelet concentrates can induce the proliferation of the dermal papilla and the vascularization of the perifollicular tissue, as well as accelerate the telogen-to-anagen transition, thereby promoting the regrowth of hair improving the appearance of hair loss. Herein, we report on the application of a new, modified form of platelet concentrates, namely, concentrated growth factors (CGFs), in 15 cases of androgenetic alopecia (AGA). 15 cases of androgenetic alopecia were treated with the use of monthly, subcutaneous injections of autologous CGF in the scalp. A total of 3 injections were administered 4 weeks apart, and the patients were followed up for 6 months. Assessments were performed before the treatments and at 4, 8, 12 and 24 weeks after the first treatment. The treatment outcomes were assessed by taking macroscopic photographs and trichoscopic photomicrographs, as well as by using the Global Aesthetic Improvement Scale (GAIS) and the patient satisfaction survey. In order to determine the safety of the treatment, the injection area was observed for signs of infection or mass evaluation. The photographs showed significant improvement in hair appearance after injections of CGF. The hair photomicrographs showed that CGF promoted the regrowth of hair in balding areas, with an increased hair density and an increased ratio of terminal to vellus hair. The GAIS suggested that CGF treatments were effective in treating AGA, and the majority of patients were satisfied with their improvement. In addition, treatments resulted in a faster rate of hair growth and a decrease in the greasy and unpleasant sensation of the hair of the patients. At the last visit, none of the 15 patients reported experiencing side-effects during the follow-up period. To conclude, the application of CGF can be an effective method in the treatment of androgenetic alopecia. 


\section{INTRODUCTION}

Androgenetic alopecia (AGA) is a major cause of hair loss, which is characterized by a progressive hair loss; AGA affects both genders, with a pattern of recession of the frontal hairline and a hair loss of the vertex in males and a pattern of central region thinning with a preserved frontal hair line in females [1]. Several studies have revealed that the pathogenesis of AGA is related to an impaired perifollicular vascularization and an imbalance of growth factors. In vitro and vivo experiments also confirmed that growth factors are involved in the regulation of hair follicle growth and development and hair cycle, such as vascular endothelial growth factor (VEGF), insulin-like growth factor (IGF-1), and fibroblast growth factor (FGFs) [2-5]. Thus, platelet concentrates can be defined as autologous concentrations of platelet-derived growth factors in the plasma [6] that are released by activated platelets after centrifugation, with these factors promoting angiogenesis, cell proliferation and differentiation in the processes of soft- and hard-tissue wound healing [7-10]. Moreover, various studies have shown that platelet concentrates can induce the proliferation of the dermal papilla, prolong the anagen phase and increase the vascularization around hair follicles, thus leading to a positive trend in hair growth $[11,12]$. These findings have been further verified in clinical trials [13].

CGF is a new type of platelet concentrate [14] that can activate platelets by variations in high-speed centrifugation without the administration of exogenous thrombin. CGF can also be easily prepared with a single centrifugal spin, which results in the product not being exposed to the environment. CGF has a good effect on promoting the vascularization of tissue as CGF contains many more angiogenesis growth factors, such as Vascular Endothelial Growth Factor (VEGF), Platelet-Derived Growth Factor-BB (PDGF-BB) and Transforming Growth Factor Beta 1 (TGF- $\beta 1$ ) $[15,16]$ which will be benefit to activate resting hair follicular stem cells, as well as having CD34 positive cells within the fibrin matrix [17], which have been shown and promising treatment candidates for the management of AGA.

However, no clinical study focusing on CGF-treated AGA has been attempted. Hence, we examined 15 cases of a novel, clinical application of autologous liquid CGF in AGA and verified the efficacy and safety of the treatments.

\section{METHODS}

\subsection{Patients}

We recruited patients who had been diagnosed with AGA for more than a year and who had hair loss types of Ludwig II-III or Norwood-Hamilton II-V. Patients who had taken finasteride or minoxidil treatments within six months before the study were excluded from the study. Other exclusion criteria included abnormal decreases in platelet counts, a history of allergies, a diagnosis of diabetes, a lack of energy-based procedures, a haematologic disease, a hepatic disease, a renal disease, a history of smoking within six months of the study and treatments with an anti-aggregating therapy.

The study was performed in accordance with the principles of the Declaration of Helsinki and was approved by the Shanghai Ninth People's Hospital Ethics Committee. All of the treatments and follow ups were performed in the Shanghai Ninth People's Hospital.

\subsection{CGF Preparation}

A 9-ml venous blood sample was drawn into a Vacuette vacuum blood collection tube (Greiner Bio-One $\mathrm{GmbH}$, Kremsmünster, Austria) that contained sodium heparin anticoagulant and was then immediately centrifuged with programmed varying speeds [15] via a Medifuge centrifugation system (Silfradent S.R.L., Santa Sofia, Italy). After centrifugation, the whole blood was divided into three layers, with the bottom layer containing the red blood cells (RBC), the overlying buffy coat (BC) containing white leukocytes, and the yellow plasma layer containing CGFs and platelet-poor plasma (PPP). Due to the factors and small molecular densities, the collection of plasma from the border of the buffy coat and the harvesting of $2-3 \mathrm{ml}$ of plasma corresponded to the CGFs (Figure 1(A)). The volume collection method that was 

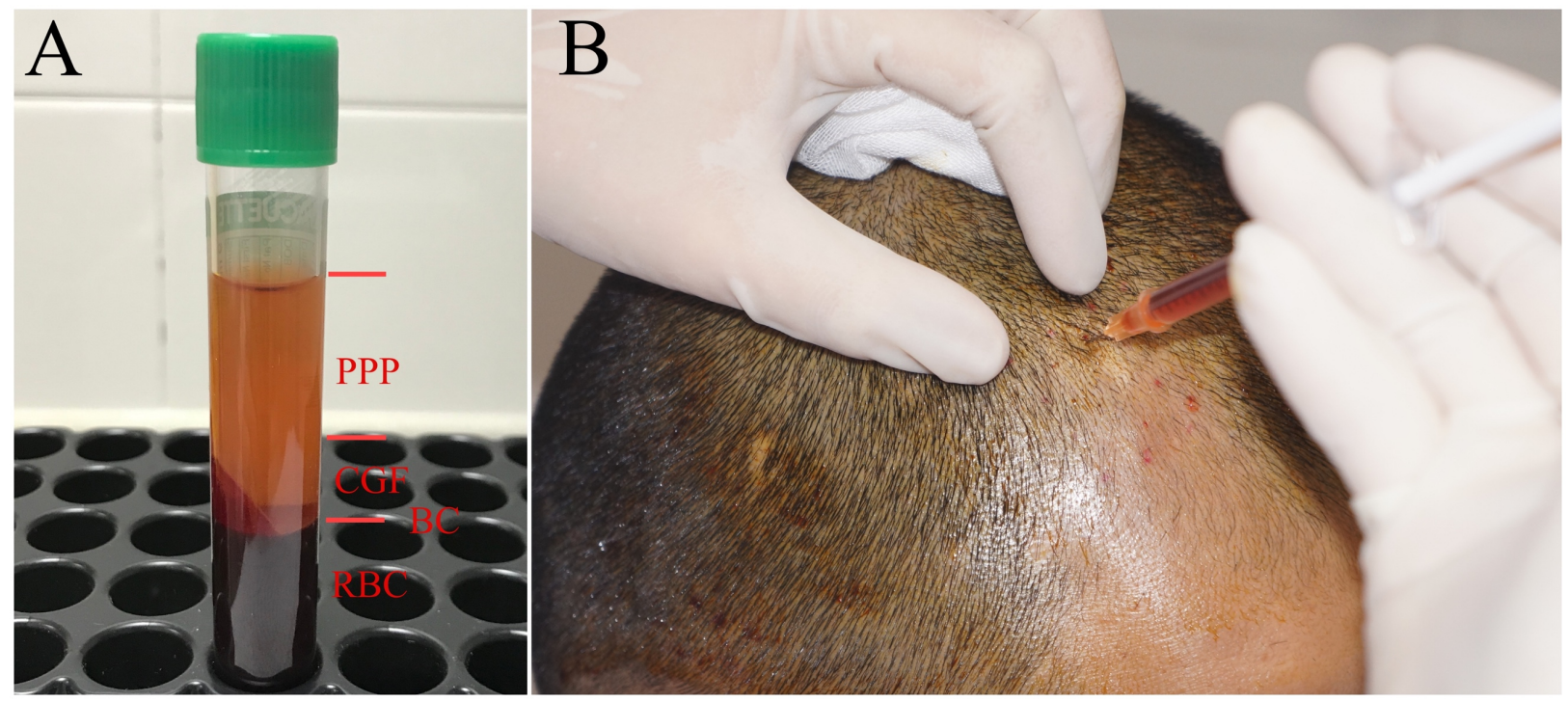

Figure 1. (A) The whole blood after being centrifuged and divided into three layers. The bottom layer was comprised of the red blood cells (RBC), the white leukocytes were found in the buffy coat (BC) above and the yellow plasma layer comprised CGF and the platelet-poor plasma (PPP); (B) CGF was subcutaneously injected, with a droplet of $0.02 \mathrm{ml}-0.05 \mathrm{ml} / \mathrm{cm}^{2}$, onto the hairless area.

previously described, with 2 - $3 \mathrm{ml} \mathrm{CGF} \mathrm{collected} \mathrm{from} \mathrm{every} 9 \mathrm{ml}$ of whole blood, increased the platelet counts 3 - to 5 -fold over the baseline concentration [18].

\subsection{Treatment and Follow Up}

Before injection, we used $2 \%$ lidocaine as supraorbital and supratrochlear nerve block anaesthetics. CGF was subcutaneously injected by using a 1-ml syringe with a 30-gauge needle in the galea aponeurotica of the frontal or parietal area, according to the range of the hairless area, and this amount was injected in a droplet of $0.05 \mathrm{ml} / \mathrm{cm}^{2}$ (Figure 1(B)).

The patients received three injections, at intervals of 4 weeks, and were followed-up for 24 weeks. The treated areas were photographed by using a digital camera and the VISIA Complexion Analysis System (Canfield, New Jersey, USA).

\subsection{Outcome Evaluation}

Hair status was assessed for a period of 24 weeks, beginning at baseline ( $1^{\text {st }}$ visit and $1^{\text {st }}$ treatment $)$ and ranging to 4 weeks ( $2^{\text {nd }}$ visit and $2^{\text {nd }}$ treatment), 8 weeks ( $3^{\text {rd }}$ visit and $3^{\text {rd }}$ treatment), 12 weeks ( $4^{\text {th }}$ visit, as the end point for effective assessment) and 24 weeks (the last visit and the end point for safety assessment), with the use of the same hair style and photographic position for each patient. The treatment outcomes were assessed by taking macroscopic photographs and trichoscopic photomicrographs, as well as by using the Global Aesthetic Improvement Scale (GAIS) (Table 1) and the patient satisfaction survey. We used the VISIA system in combination with high-definition digital photography. Trichoscopic photomicrographs were used to observe the hair in micrographs and to count the number of hairs, as well as to measure the hair density and terminal to vellus hair ratios ( $\mathrm{T} / \mathrm{V}$ ratio). All of the photographs were compared with the baseline photos by an independent doctor (YX), according to the Global GAIS, and the patients were also self-evaluated by the use of the patient satisfaction survey (-1: unsatisfied; 1 : satisfied; 2 : very satisfied).

All injection site reactions, such as redness, tenderness and pain, were recorded as adverse events and were assessed by the masked investigators for the severity and relationship to the treatment from baseline to 24 weeks. 
Table 1. The global aesthetic improvement scale (GAIS) of the efficacy evaluation for five degrees.

\begin{tabular}{|c|c|c|}
\hline Score & State & Description \\
\hline 3 & Very much improved & Optimal aesthetic result for the treatment in this patient. \\
\hline 2 & Much improved & $\begin{array}{l}\text { Marked improvement in hair appearance, but not completely optimal, } \\
\text { and a touch-up would improve the result. }\end{array}$ \\
\hline 1 & Improved & $\begin{array}{l}\text { Improvement in hair appearance from the original condition, but a } \\
\text { touch-up is advised. }\end{array}$ \\
\hline 0 & No change & $\begin{array}{l}\text { The hair appearance is essentially the same compared with the original } \\
\text { condition. }\end{array}$ \\
\hline-1 & Worse & The hair appearance is worse than the original condition. \\
\hline
\end{tabular}

\subsection{Statistical Analysis}

GraphPad Prism 7 (GraphPad, San Diego, USA) was used for statistical analyses of the data. The results of the measured evaluations were expressed as the mean \pm standard deviation (SD), and the differences of hair density and T/V ratio were assessed by using two-tailed, paired sample t-tests. $\mathrm{P}$-values $<0.05$ were considered to be statistically significant.

\section{RESULTS}

From February 2017 to March 2018, a total of 15 AGA patients (6 females and 9 males) were enrolled in the study. The history of hair loss among the subjects was $2-10$ years. Blood examinations showed normal levels of platelet counts for all patients, except for a female patient (case 15) with mild, low-haemoglobin levels. The baseline characteristics are shown in Table 2.

When comparing the photographs taken before and after 12 weeks of treatment, CGF treatment led to significantly improved hair appearances. The hair photomicrographs showed that CGF treatment promoted the regrowth of hair in balding areas, with increases in hair density and ratios of terminal to vellus hair. The mean of the hair density was $40.8 \pm 17.4 / \mathrm{cm}^{2}$ at baseline and increased to $50.0 \pm 20.9 / \mathrm{cm}^{2}$ at 12 weeks (Figure 2(A)), and the T/V ratio was $47.1 \% \pm 17.3 \%$ at baseline and increased to $80.2 \% \pm 43.6 \%$ at 12 weeks (P-value < 0.05) (Figure 2(B)). The mean GAIS score was ranked at 12 weeks and 24 weeks by the independent doctor was $1.20 \pm 0.68$ (12 weeks) and $1.00 \pm 0.76$ (24 weeks), and by patients that was $1.33 \pm 0.72$ (12 weeks) and $0.80 \pm 0.68$ (24 weeks) (P-value > 0.05), but none of the patients worsened, compared to the baseline data (Figure 3), which suggested that the CGF treatments were effective. 6 patients (40\%) were very satisfied with their improvement, and 7 patients $(47 \%)$ were satisfied with their improvement, but 2 patients (13\%) were unsatisfied. In addition, 8 patients (53\%) reported that the CGF treatments resulted in faster rates of hair growth and decreases in the greasy texture of the hair, as well as decreases in itching or burning sensations, for one to two weeks after the injections.

At 24 weeks, 11 cases (73\%) had no relapses, and 4 patients (27\%) exhibited relapses. All 15 cases exhibited no adverse effects, but the patients complained of slight pain during the injection procedure; however, this pain was spontaneously resolved afterward.

\subsection{Case 1}

A 27-year-old female patient had Ludwig III AGA and hair loss for 5 years. The pictures showed that her hair appearance was improved after three injections (Figure 4).

\subsection{Case 2}

A 27-year-old male patient had Hamilton-Norwood IVa AGA and hair loss for 6 years. The pictures showed that his hair appearance was improved after three injections (Figure 5). 
Table 2. Characteristics of the study patients.

\begin{tabular}{ccccc}
\hline No & Gender & Age & The history of AGA (years) & Hair loss type \\
\hline 1 & Female & 27 & 5 & Ludwig III \\
2 & Male & 44 & 13 & Norwood-Hamilton Va \\
3 & Male & 27 & 6 & Norwood-Hamilton IVa \\
4 & Male & 35 & 7 & Norwood-Hamilton IV \\
5 & Female & 25 & 2 & Ludwig II \\
6 & Male & 30 & 4 & Norwood-Hamilton III \\
7 & Male & 28 & 3 & Norwood-Hamilton II \\
8 & Female & 21 & 2 & Ludwig I \\
9 & Female & 29 & 4 & Ludwig III \\
10 & Male & 24 & 2 & Norwood-Hamilton II \\
11 & Male & 36 & 8 & Norwood-Hamilton IV \\
12 & Female & 34 & 2 & Ludwig II \\
13 & Male & 30 & 5 & Norwood-Hamilton III \\
14 & Male & 42 & 10 & Norwood-Hamilton V \\
15 & Female & 48 & 2 & Ludwig I \\
\hline
\end{tabular}

Hair Density

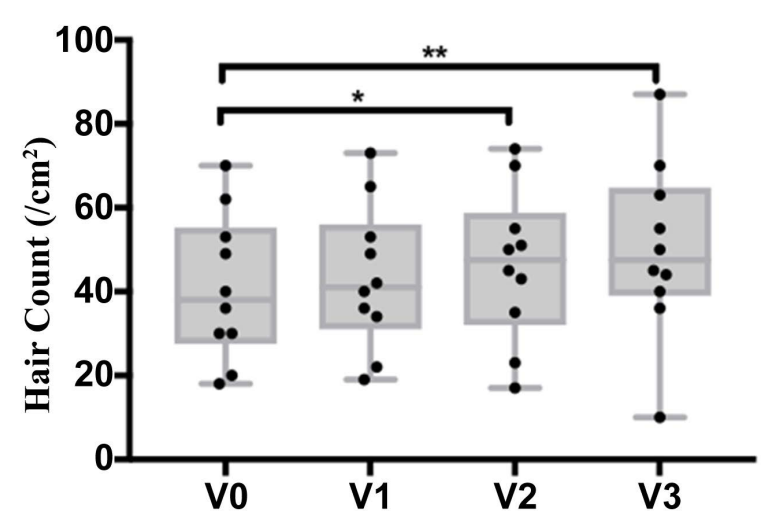

Follow-up

(A)

\section{T/V ratio}

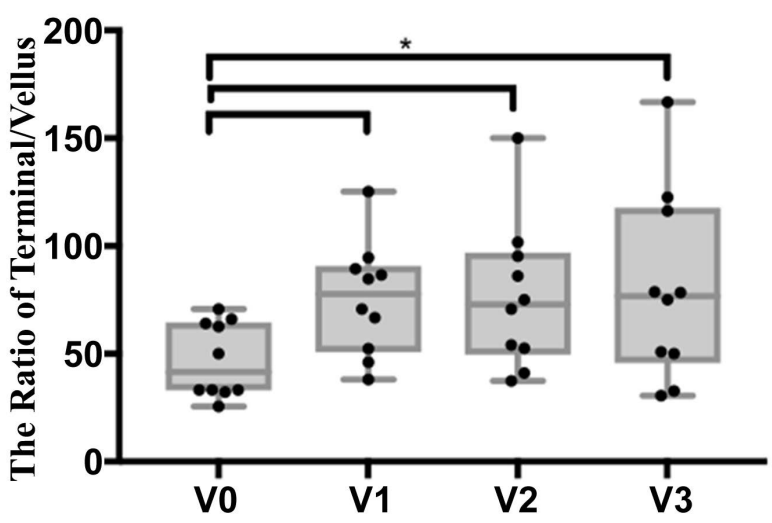

Follow-up

(B)

Figure 2. Compared with hair photomicrographs to count hair density (A) and the $\mathrm{T} / \mathrm{V}$ ratio (B) from baseline (V0) to 12 weeks (V3) $\left({ }^{*}, \mathrm{P}\right.$-value $<0.05 ;{ }^{* *}$, P-value $\left.<0.01\right)$.

\section{DISCUSSION}

Androgenic alopecia is a sex-hormone mediated hair loss condition. The prevalence of androgenetic alopecia increases with age, varies among different races and is less common among Asian than Caucasian patients. Accordingly, the incidence of this condition affects up to $80 \%$ of Caucasian men and $42 \%$ of women [19]; furthermore, in China, this incidence was reported to be $21.3 \%$ in men and $6.0 \%$ in women. The main cause of AGA is abnormal amounts of the metabolite dihydrotestosterone (DHT), which is a 
GAIS score at 12 weeks

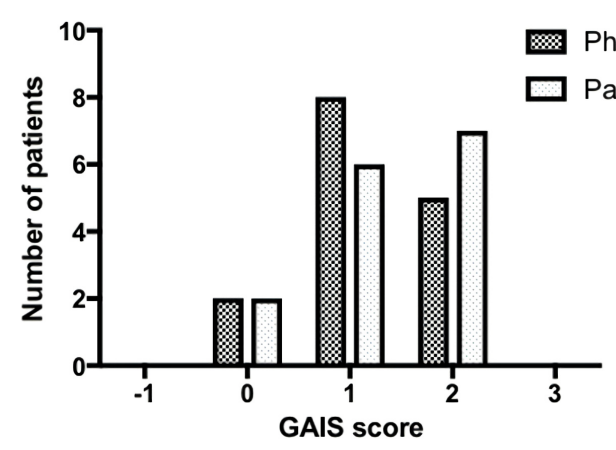

(A)

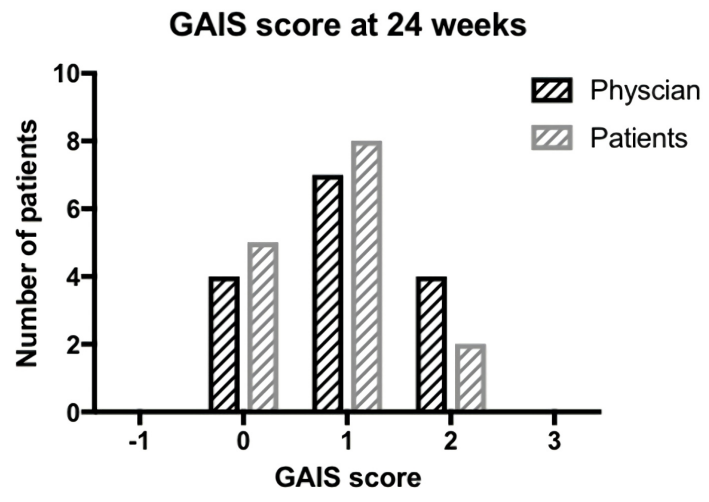

(B)

Figure 3. The statistical graphs of GAIS score was evaluated by (A) physician and (B) patients at 12 weeks (V3) and 24 weeks (V4).
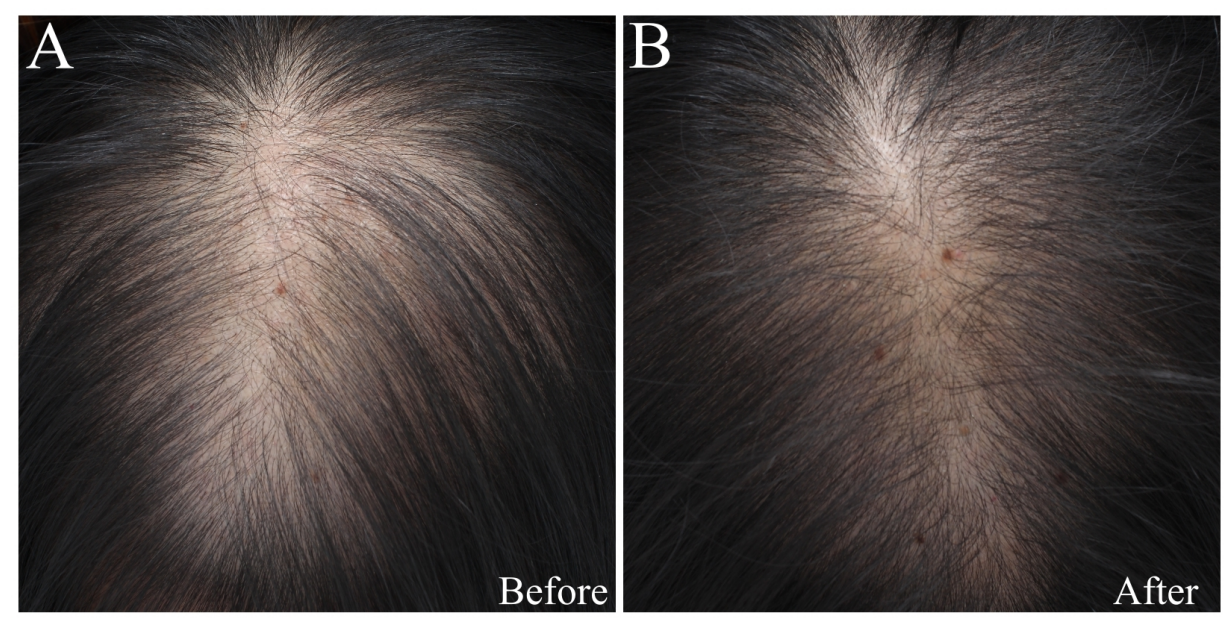

Figure 4. A 27-year-old female patient with Ludwig II AGA (case 1) (A) before treatment (V0) and (B) after three injections at 12 weeks (V3).
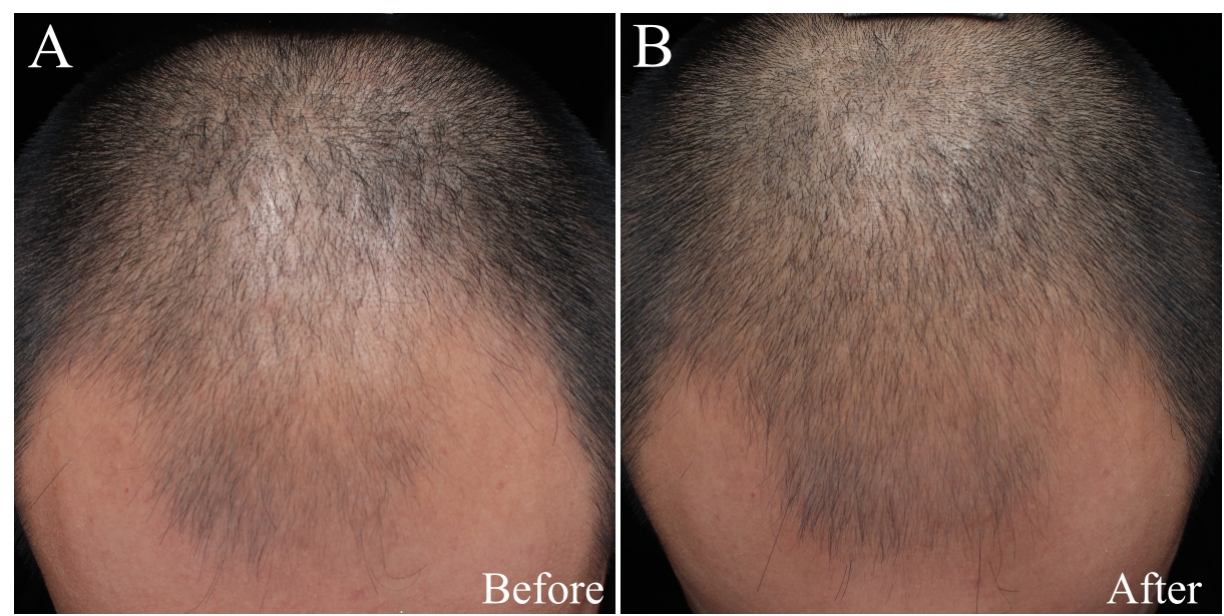

Figure 5. A 27-year-old male patient with Hamilton-Norwood IIIa AGA (case 2) (A) before treatment (V0) and (B) after three injections at 12 weeks (V3). 
primary androgen that is converted by 5-alpha-reductase from testosterone, and the genetically determined sensitivity of the androgen receptors on the hair follicles also increases exposure to serum DHT [20]. DHT contributes to several pathological changes, including the catagenic miniaturization of the hair follicles, a shortened anagen period and an extended telogen period, after which the terminal hair is replaced by a soft vellus [21]. DHT also contributes to the presence of dermal papillae (DP) at different sites, with these papillae having completely opposite effects, including the stimulation of facial beard growth by an increase in the production of IGF-1 from beard DP cells as positive mediators. Additionally, an inhibition in hair growth can be stimulated at the temples and scalp apex in AGA via releases of TGF- $\beta 1$, TGF- $\beta 2$ and IL- 6 from the bald DP cells as negative mediators [22].

Androgenetic alopecia has been treated in several ways, but current available treatments seem to be limited by adverse reactions or low efficiency [23]. Follicular unit transplantation (FUT) is a surgical procedure that involves the transplantation of hair follicles from the occipital region of the donor to the balding area of the recipient, but the procedure is invasive and can result in pain and bleeding, with the survival of the donor follicle units being dependent on the operative and technological expertise of the surgeon. Other non-surgical products have been proposed as therapies for alopecia, with the most common therapies that have been approved by the Food and Drug Administration(FDA) including oral finasteride (for treatment in males), as well as topical minoxidil and low-level laser treatment in both males and females. Finasteride is a selective inhibitor of type II 5 alpha reductase, which results in the inhibition of the conversion of testosterone into DHT and the reduction of the androgen-dependent miniaturization of the hair follicles [24]. However, male patients have been reported to refuse these treatments because finasteride can increase the risk of erectile dysfunction; additionally, the FDA published a warning that the 5 - $\alpha$-reductase inhibitor class of drugs may increase the risk of prostate cancer [25]. The mechanism of minoxidil is as a potassium channel opener that increases blood supply to the hair follicles and inhibits the apoptosis of the dermal papilla, but the main adverse effects involve facial hypertrichosis and itching, redness or irritation to the applied area. Low-level laser therapy is a relatively new treatment that was approved by the FDA for the treatment of AGA. It has been proposed that the light energy activates cellular signalling and increases electron transport, in order to promote the regrowth of hair. However, there is a lack of high-level evidence from clinical trials, which is needed to establish the efficacy and safety of the use of low-level laser therapy $[21,26]$.

To explore the use of future therapies for AGA, various studies have verified that the vascularity of the hair follicles is closely related to hair growth. In 2001, Mecklenburg reported that the increased perifollicular vascular formation during the anagen phase and hair follicular vascularization promote hairs to transition from the telogen phase to the anagen phase by inhibiting vascularity, which can hinder hair growth [27]. Goldman et al. also demonstrated that VEGF mainly induces vascular perifollicular formation, promotes hair growth and enlarges the size of the hair follicles, and they also demonstrated that the expression of VEGF was reduced in patients with AGA [28]. In fact, DP cells secrete a variety of growth factors, such as Epidermal Growth Factor (EGF), Fibroblast Growth Factor (FGF) and Insulin-Like Growth Factor Type 1 (IGF-1), which are involved in the regulation of hair morphogenesis and the hair growth cycle. A study reported by Akiyama et al. demonstrated that GFs are involved in regulating the growth and differentiation of bulge cells and that Platelet-Derived Growth Factors (PDGFs) may have related roles in the interaction between the bulge cells and the associated tissue during follicle morphogenesis [2]. The application of bFGF to the back skin of mice can enhance the size of the hair follicles and can affect the hair growth cycle [4]. In addition, Zhao J. et al. also illustrated that DHT inhibits sensory neuronal stimulation by interacting with androgen receptors, thereby decreasing the production of IGF-1 in the dermal papilla and inhibiting hair growth in in vitro experiments [29]. Further studies have revealed that exogenous IGF-1 promotes hair growth by stimulating the proliferation of follicle cells in the anagen phase of the matrix and by inhibiting the expression of TGF- $\beta 1$ in the hair follicles [5].

From this view, platelet concentrates that enrich high concentrations of platelets after centrifugation may provide many types of platelet-derived growth factors [6], which can promote angiogenesis and the regeneration of tissue [7-10]. Platelet-rich plasma (PRP) has been the most commonly used type of platelet 
concentrate, and the use of PRP in AGA has been investigated in numerous clinical studies focusing on hair loss, but the results have been controversial. Until 2017, a meta-analysis suggested that the use of PRP for the treatment of AGA was effective [30], as well as useful for stimulating hair growth by promoting vascularization and prolonging the anagen stage [12]. Multiple PRP-derived growth factors can mediate the activation of wingless (Wnt) $\beta$-catenin, extracellular signalling regulated kinase (ERK) and protein kinase B (Akt) signalling pathways, thereby increasing cell proliferation and differentiation, as well as inhibiting apoptosis and prolonging cellular survival [12]. Similar evidence has been presented by Li et al., who found that PRP increased the proliferation of DP cells and stimulated ERK and Akt signalling, as well as increasing the expression of fibroblast growth factor 7 (FGF-7) and $\beta$-catenin for the acceleration of the telogen-to-anagen transition in an in vitro model [31].

Via the modification of the preparation of platelet concentrates, a new form defined as concentrated growth factors (CGF) was extracted with a variable speed centrifugation [14]. The preparation of these concentrates is simpler and more convenient, involving only a single centrifugation of 13 mins without the need to add exogenous activators. Compared with PRP, CGF contains higher levels of platelets and platelet-derived growth factors, such as TGF- $\beta 1$, VEGF and PDGF-BB, which are characterized by promoting angiogenesis [15, 32]. Rodella et al. also demonstrated the existence of CD34 positive cells in CGF. CGF stimulates the cell growth, proliferation and metabolic activity of many different cell types in vitro [33], and CGF has also increased graft angiogenesis and wound healing around the sites of implants in vivo [34, 35]. Masuki et al. found that the platelet-rich fibrin clot exudates resulted in reduced levels of growth factors [15]. Therefore, we speculate that the effective ingredient in CGF can be obtained in liquid form, if prepared with anticoagulation and injected with CGF, in order to directly deliver the growth factors to the bald scalp.

Our study is the first to use CGF for the treatment of AGA in the Asian population. This is also the first report of a CGF injection in liquid form. In our study, CGF was found to significantly promote the regrowth of hair, to increase hair coverage and to improve hair appearance on the scalp. Furthermore, these cases showed that CGF could reduce the greasy feeling of hair, as well as reducing the itching and burning sensations of the scalp, by regulating the secretions of sebaceous glands. At the last visit of the follow-up period, the new hairs grew into fully pigmented terminal hairs, and the majority of the patients had no relapses after the two months from the last injections. No localized or systematic side effects occurred. The patients complained of pain during the injection process, which reduced patient satisfaction and compliance, which is an important issue for the improvement of treatments. The lack of a control group and a long-term follow-up period also limits the importance of our findings.

\section{CONCLUSION}

To conclude, our investigation demonstrated the effectiveness and safety of CGF in the treatment of female and male AGA patients. CGF, with no need for the addition of exogenous activators, could be a promising therapy for AGA. Further research with larger sample sizes is still required, in order to determine the long-term efficacy of the injection of CGF in AGA.

\section{ACKNOWLEDGEMENTS}

This research was supported by the National Natural Science Foundation of China (No. 81501678; No. 81620108019).

\section{AUTHOR CONTRIBUTION}

PCT and YX initiated and designed the study and protocol. WW, PQZ and YMG participated in data collection. PCT analyzed the data. YMG and PQZ contributed in data interpretation. PCT and YX wrote the first draft of the manuscript, and QFL, SBZ and KL critiqued and modified the manuscript. All authors reviewed and approved the work. 


\section{AUTHOR DISCLOSURE STATEMENT}

None of the authors has a financial interest in any of the products, devices, or drugs mentioned in this manuscript.

\section{CONFLICTS OF INTEREST}

The authors declare no conflicts of interest regarding the publication of this paper.

\section{REFERENCES}

1. Blumeyer, A., Tosti, A., Messenger, A., Reygagne, P., Del Marmol, V., Spuls, P.I., Trakatelli, M., Finner, A., Kiesewetter, F., Trueb, R., Rzany, B. and Blume-Peytavi, U. (2011) Evidence-Based (S3) Guideline for the Treatment of Androgenetic Alopecia in Women and in Men. Journal der Deutschen Dermatologischen Gesellschaft, 9, 51-57. https://doi.org/10.1111/j.1610-0379.2011.07802.x

2. Akiyama, M., Smith, L.T. and Holbrook, K.A. (1996) Growth Factor and Growth Factor Receptor Localization in the Hair Follicle Bulge and Associated Tissue in Human Fetus. Journal of Investigative Dermatology, 106, 391-396. https://doi.org/10.1111/1523-1747.ep12343381

3. Yano, K., Brown, L.F. and Detmar, M. (2001) Control of Hair Growth and Follicle Size by VEGF-Mediated Angiogenesis. Journal of Clinical Investigation, 107, 409-417. https://doi.org/10.1172/JCI11317

4. 4zeki, M. and Tabata, Y. (2002) Promoted Growth of Murine Hair Follicles through Controlled Release of Basic Fibroblast Growth Factor. Tissue Engineering, 8, 359-366. https://doi.org/10.1089/107632702760184637

5. Li, J., Yang, Z., Li, Z., Gu, L., Wang, Y. and Sung, C. (2014) Exogenous IGF-1 Promotes Hair Growth by Stimulating Cell Proliferation and Down Regulating TGF-Beta1 in C57BL/6 Mice in Vivo. Growth Hormone \& IGF Research 24, 89-94. https://doi.org/10.1016/j.ghir.2014.03.004

6. Eppley, B.L., Woodell, J.E. and Higgins, J. (2004) Platelet Quantification and Growth Factor Analysis from Platelet-Rich Plasma: Implications for Wound Healing. Plastic and Reconstructive Surgery, 114, 1502-1508. https://doi.org/10.1097/01.PRS.0000138251.07040.51

7. Mahindra, P., Yamin, M., Selhi, H.S., Singla, S. and Soni, A. (2016) Chronic Plantar Fasciitis: Effect of Platelet-Rich Plasma, Corticosteroid, and Placebo. Orthopedics, 39, e285-289. https://doi.org/10.3928/01477447-20160222-01

8. Castillo-Cardiel, G., Medina-Quintana, V.M., Lomeli-Enriquez, M., Medrano-Munoz, F., Guerrero-Velazquez, C., Contreras-Lopez, C.K., Fuentes-Orozco, C., Irusteta-Jimenez, L., Michel-Espinoza, L.R. and Gonzalez-Ojeda, A. (2017) Platelet-Rich Plasma and Its Effect in Bone Regeneration in Mandibular Fractures. Controlled Clinical Trial. Gaceta Medica De Mexico, 153, 459-465.

9. Suthar, M., Gupta, S., Bukhari, S. and Ponemone, V. (2017) Treatment of Chronic Non-Healing Ulcers Using Autologous Platelet Rich Plasma: A Case Series. Journal of Biomedical Science, 24, 16. https://doi.org/10.1186/s12929-017-0324-1

10. Stavrakas, M., Karkos, P.D., Markou, K. and Grigoriadis, N. (2016) Platelet-Rich Plasma in Otolaryngology. The Journal of Laryngology \& Otology, 130, 1098-1102. https://doi.org/10.1017/S0022215116009403

11. Shen, H., Cheng, H., Chen, H. and Zhang, J. (2017) Identification of Key Genes Induced by Platelet-Rich Plasma in Human Dermal Papilla Cells Using Bioinformatics Methods. Molecular Medicine Reports, 15, 81-88. https://doi.org/10.3892/mmr.2016.5988

12. Gupta, A.K. and Carviel, J. (2016) A Mechanistic Model of Platelet-Rich Plasma Treatment for Androgenetic Alopecia. Dermatologic Surgery, 42, 1335-1339. https://doi.org/10.1097/DSS.0000000000000901

13. Giordano, S., Romeo, M. and Lankinen, P. (2017) Platelet-Rich Plasma for Androgenetic Alopecia: Does It 
Work? Evidence from Meta Analysis. Journal of Cosmetic Dermatology, 16, 374-381.

https://doi.org/10.1111/jocd.12331

14. Sacco, L.L. (2006) International Academy of Implant Prosthesis and Osteoconduction.

15. Masuki, H., Okudera, T., Watanebe, T., Suzuki, M., Nishiyama, K., Okudera, H., Nakata, K., Uematsu, K., Su, C.Y. and Kawase, T. (2016). Growth Factor and Pro-Inflammatory Cytokine Contents in Platelet-Rich Plasma (PRP), Plasma Rich in Growth Factors (PRGF), Advanced Platelet-Rich Fibrin (A-PRF), and Concentrated Growth Factors (CGF). International Journal of Implant Dentistry, 2, 19.

https://doi.org/10.1186/s40729-016-0052-4

16. Isobe, K., Watanebe, T., Kawabata, H., Kitamura, Y., Okudera, T., Okudera, H., Uematsu, K., Okuda, K., Nakata, K., Tanaka, T. and Kawase, T. (2017) Mechanical and Degradation Properties of Advanced Platelet-Rich Fibrin (A-PRF), Concentrated Growth Factors (CGF), and Platelet-Poor Plasma-Derived Fibrin (PPTF). International Journal of Implant Dentistry, 3, 17. https://doi.org/10.1186/s40729-017-0081-7

17. Rodella, L.F., Favero, G., Boninsegna, R., Buffoli, B., Labanca, M., Scari, G., Sacco, L., Batani, T. and Rezzani, R. (2011) Growth Factors, CD34 Positive Cells, and Fibrin Network Analysis in Concentrated Growth Factors Fraction. Microscopy Research and Technique, 74, 772-777. https://doi.org/10.1002/jemt.20968

18. Rattanasuwan, K., Rassameemasmaung, S., Kiattavorncharoen, S., Sirikulsathean, A., Thorsuwan, J. and Wongsankakorn, W. (2018) Platelet-Rich Plasma Stimulated Proliferation, Migration, and Attachment of Cultured Periodontal Ligament Cells. European Journal of Dentistry, 12, 469-474. https://doi.org/10.4103/ejd.ejd_255_17

19. Kanti, V., Messenger, A., Dobos, G., Reygagne, P., Finner, A., Blumeyer, A., Trakatelli, M., Tosti, A., Del Marmol, V., Piraccini, B.M., Nast, A. and Blume-Peytavi, U. (2018) Evidence-Based (S3) Guideline for the Treatment of Androgenetic Alopecia in Women and in Men-Short Version. Journal of the European Academy of Dermatology and Venereology, 32, 11-22. https://doi.org/10.1111/jdv.14624

20. Wang, T.L., Zhou, C., Shen, Y.W., Wang, X.Y., Ding, X.L., Tian, S., Liu, Y., Peng, G.H., Xue, S.Q., Zhou, J.E., Wang, R.L., Meng, X.M., Pei, G.D., Bai, Y.H., Liu, Q., Li, H. and Zhang, J.Z. (2010) Prevalence of Androgenetic Alopecia in China: A Community-Based Study in Six Cities. British Journal of Dermatology, 162, 843-847. https://doi.org/10.1111/j.1365-2133.2010.09640.x

21. Rossi, A., Anzalone, A., Fortuna, M.C., Caro, G., Garelli, V., Pranteda, G. and Carlesimo, M. (2016) Multi-Therapies in Androgenetic Alopecia: Review and Clinical Experiences. Dermatologic Therapy, 29, 424-432. https://doi.org/10.1111/dth.12390

22. Inui, S. and Itami, S. (2013) Androgen Actions on the Human Hair Follicle: Perspectives. Experimental Dermatology, 22, 168-171. https://doi.org/10.1111/exd.12024

23. Jain, R. and De-Eknamkul, W. (2014) Potential Targets in the Discovery of New Hair Growth Promoters for Androgenic Alopecia. Expert Opinion on Therapeutic Targets, 18, 787-806. https://doi.org/10.1517/14728222.2014.922956

24. FDA Drug Safety Communication: 5-Alpha Reductase Inhibitors (5-ARIs) May Increase the Risk of a More Serious Form of Prostate Cancer.

25. Mella, J.M., Perret, M.C., Manzotti, M., Catalano, H.N. and Guyatt, G. (2010) Efficacy and Safety of Finasteride Therapy for Androgenetic Alopecia: A Systematic Review. Archives of Dermatology, 146, 1141-1150. https://doi.org/10.1001/archdermatol.2010.256

26. Varothai, S. and Bergfeld, W.F. (2014) Androgenetic Alopecia: An Evidence-Based Treatment Update. American Journal of Clinical Dermatology, 15, 217-230. https://doi.org/10.1007/s40257-014-0077-5

27. Mecklenburg, L., Tobin, D.J., Muller-Rover, S., Handjiski, B., Wendt, G., Peters, E.M., Pohl, S., Moll, I. and Paus, R. (2000) Active Hair Growth (Anagen) Is Associated with Angiogenesis. Journal of Investigative Derma- 
tology, 114, 909-916. https://doi.org/10.1046/j.1523-1747.2000.00954.x

28. Goldman, C.K., Tsai, J.C., Soroceanu, L. and Gillespie, G.Y. (1995) Loss of Vascular Endothelial Growth Factor in Human Alopecia Hair Follicles. Journal of Investigative Dermatology, 104, 18S-20S. https://doi.org/10.1038/jid.1995.40

29. Zhao, J., Harada, N. and Okajima, K. (2011) Dihydrotestosterone Inhibits Hair Growth in Mice by Inhibiting Insulin-Like Growth Factor-I Production in Dermal Papillae. Growth Hormone \& IGF Research, 21, 260-267. https://doi.org/10.1016/j.ghir.2011.07.003

30. Gupta, A.K. and Carviel, J.L. (2017) Meta-Analysis of Efficacy of Platelet-Rich Plasma Therapy for Androgenetic Alopecia. Journal of Dermatological Treatment, 28, 55-58. https://doi.org/10.1080/09546634.2016.1179712

31. Li, Z.J., Choi, H.I., Choi, D.K., Sohn, K.C., Im, M., Seo, Y.J., Lee, Y.H., Lee, J.H. and Lee, Y. (2012) Autologous Platelet-Rich Plasma: A Potential Therapeutic Tool for Promoting Hair Growth. Dermatologic Surgery, 38, 1040-1046.

32. Qiao, J., An, N. and Ouyang, X. (2017) Quantification of Growth Factors in Different Platelet Concentrates. Platelets, 28, 774-778. https://doi.org/10.1080/09537104.2016.1267338

33. Bonazza, V., Borsani, E., Buffoli, B., Parolini, S., Inchingolo, F., Rezzani, R. and Rodella, L.F. (2018) In Vitro Treatment with Concentrated Growth Factors (CGF) and Sodium Orthosilicate Positively Affects Cell Renewal in Three Different Human Cell Lines. Cell Biology International, 42, 353-364. https://doi.org/10.1002/cbin.10908

34. Hu, Y., Jiang, Y., Wang, M., Tian, W. and Wang, H. (2018) Concentrated Growth Factor Enhanced Fat Graft Survival: A Comparative Study. Dermatologic Surgery, 44, 976-984. https://doi.org/10.1097/DSS.0000000000001337

35. Sahin, I.O., Gokmenoglu, C. and Kara, C. (2018) Effect of Concentrated Growth Factor on Osteoblast Cell Response. Journal of Stomatology, Oral and Maxillofacial Surgery, 119, 477-481.

https://doi.org/10.1016/j.jormas.2018.06.001 